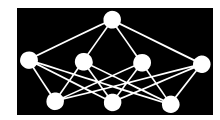

\title{
MULTI USER DETECTION USING FUZZY LOGIC EMPOWERED ADAPTIVE BACK PROPAGATION NEURAL NETWORK
}

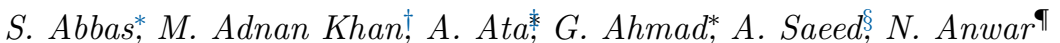

\begin{abstract}
In Wireless communication, Multiple Input and Multiple Output (MIMO) systems have always been quite popular. Multicarrier systems are established along with different techniques of space-time coding to accomplish the demands of these systems. One of the most popular techniques is Multi-Carrier Code Division Multiple Access (MC-CDMA) with Alamouti's Space-Time Block Codes (STBC). This article, proposed the Fuzzy Logic empowered Adaptive Back Propagation Neural Network (FLeABPNN) based Multi User Detection (MUD) system, which is used to determine the receiver weights of MC-CDMA with the scheme of two variations. The proposed FLeABPNN approach takes advantage of a neuro-fuzzy hybrid system which conglomerates the competences of both fuzzy logic and neural networks for multi-user detection. It is observed that due to the fuzzy logic-based learning rate, proposed FLeABPNN based receiver without relationship \& with relationship achieved the $3.04 \times 10^{-06}$ and $2.05 \times 10^{-06}$ Bit Error Rate (BER) respectively. The proposed FLeABPNN based receiver gives fast convergence rate \& low BER as compared to other suboptimal published techniques like GA \& LMS. It also observed that the Computational Complexity of the proposed FLeABPNN based MC-CDMA receiver is less then LMS based receiver up to 18 users, but higher than GA based receiver.
\end{abstract}

Key words: $M C-C D M A, F L e A B P N N, M I M O, B E R, A N N, S T B C, M M S E, G A$, $L M S$

Received: January 22, 2019

DOI: $10.14311 / \mathrm{NNW} .2019 .29 .024$

Revised and accepted: December 30, 2019

\section{Introduction}

Multiple access techniques are very popular when it comes to real-time wireless communication systems. There are numerous varieties of multiple access techniques are Time Division Multiple Access (TDMA), Frequency Division Multiple

\footnotetext{
*Sagheer Abbas, Gulzar Ahmad; School of Computer Science, NCBA\&E, Lahore, E-Mail: dr.sagheer@ncbae.edu.pk, Gulzar.phd@ncbae.edu.pk

${ }^{\dagger}$ Muhammad Adnan Khan - corresponding author; Department of Computer Science, Lahore Garrison University, Lahore, E-Mail: madnankhan@lgu.edu.pk

$\ddagger_{A}$. Ata; School of Computer Science, NCBA\&E, Lahore \& Dept of Computer Science, GCU, Lahore, E-mail: ayesha.ravian@gmail.com

$\S$ Anwaar Saeed, Nida Anwar; Department of Computer Science, Virtual University, Lahore. E-Mail: anwarsaeed@vu.edu.pk, nidaanwar@vu.edu.pk
} 
Access (FDMA) \& Code Division Multiple Access (CDMA), which were designed for achieving the high data rate with a maximum number of users. TDMA allows several users to share the same frequency channel by dividing the signal into different time slots. Frequency Division Multiple Access (FDMA) allows multiple users to send data through a single communication channel, by dividing the bandwidth of the channel into separate non-overlapping frequency sub-channels and allocating each sub-channel to a separate user. Similarly, Code Division Multiple Access (CDMA) also a multiple access technology where the users are separated by unique orthogonal codes, which means that all users can use the same frequency and transmit at the same time. [1-3].

The number of users of wireless communication systems is increasing day by day. To accommodate these users, Multi-Carrier Systems like Multi-Carrier CDMA (MC-CDMA) \& Orthogonal Frequency Division Multiple Access (OFDMA) are used [4-6]. MC-CDMA is a multiple access scheme used in OFDM-based telecommunication systems, allowing the system to support multiple users at the same time over the same frequency band. Another addition to multicarrier systems is Multiple Input and Multiple Output (MIMO) that uses multiple antennas on both transmitter and receiver sides. This technology got good attention in Beyond 3rd Generation (B3G) wireless networks [7] due to the fact of maximum utilization of limited bandwidth [8], [9].

The performance of the MIMO based MC-CDMA system may suffer due to Multi-User Interference (MUI) and Near-Far Rate (NFR). To overcome these issues, different intelligent Multi-User Detection (MUD) algorithms have been proposed for MIMO based MC-CDMA systems [10]. Multi User Detection methods are classified as under:

- Blind methods

- Semi blind methods

- Training based methods

Training based methods are associated with different training symbols, which results in stable MIMO based multiuser estimation. Furthermore, the bandwidth efficiency of the system is also improved [8]. In recent years, there is an increase in the importance of training-based MIMO MUD. Four types of training-based multi-user estimators are Least Squared (LS), SLS estimator, linear MMSE channel estimator and Relaxed MMSE (RMMSE) method [11].

Blind methods; on the other hand, give high system throughput without any training streams. Various statistics based on blind multiuser detection methods have been developed. These methods can be characterized as second-order statistics \& higher-order statistics-based techniques [8]. Decision directed estimation is also a kind of blind estimation method. Similarly, numerous other blind MUD algorithms have been designed for OFDM systems in [12].

Semi blind systems are suitable for practical applications. The computational complexity of semi-blind methods is low and few training symbols have been used as compared to training-based approaches. Few training symbols have been used by semi-blind techniques to provide primary MIMO channel \& multi-user estimation $[8]$. 
Various multi-user data estimation techniques have been proposed for SpaceTime Coded (STC) based MIMO systems by using a pilot signal [13]. Orthogonal Space-Time Block Codes (OSTBs) with Maximum Likelihood (ML) decoders have achieved maximum diversity with low complexity. The ML decoder should have appropriate multi-user information so that the received signals are decoded accurately [7].

Various algorithms like ML and Zero Forcing (ZF) have been designed for symbol estimation. As compared to the ML algorithm, the implementation of the ZF algorithm is simple and less complicated. ZF algorithm has been preferred for fast fading channels. However, the ML algorithm has given better results in such environments at the cost of high computational complexity. For each subcarrier, a candidate symbol vector is examined and then Euclidean distance between the received and the original symbol is calculated for all probable arrangements of transferred symbols. Moreover, there is an exponential increase in search space with an increase in the number of transmitter and receiver antennas. As a result, the computational complexity of the system is also increased [10]. The adaptive implementation of the MIMO system is better to approach in order to overcome the computation complexity problem $[10,36,37]$.

Blind data estimation recognition has used iterative least square with projection. Moreover, heuristics approaches like those that Genetic Algorithm (GA), Pirahna Fish optimization (PFO), and Particle Swarm Optimization (PSO) are used along with ML-based multi-user data detection because these have the capability to decrease the search space and computational complexity of ML algorithms [14-19]. In MIMO based systems, the DE algorithm has also reduced the ML algorithm's search space and computational complexity of symbol detection 10, [16-19]. Apart from these, ML algorithms are designed to estimate multipath fading channel and transmitted data sequence jointly. The algorithm operates on the iterative approach and does not require any prior channel information [12,20-24].

There are numerous strategies like Neural Network [25-27,41], Genetic Algorithm (GA) [31-33] Differential Equation (DE), Cooperative Co-Evolutionary (CC) Algorithms [34], Particle Swarm Optimization (PSO) [40], Maximum Likelihood (ML) [5,6], Partial Opposite Mutant Particle Swarm Optimization (POMPSO), Total Opposite Mutant Particle Swarm Optimization (TOMPSO) [35-37], Island GA, Differential Equation (DE) and Island DE has been proposed which further enhance the performance of the 5 -th generation communication network [20,38, 39, 41, 42].

In this paper, we have proposed a new mechanism that helps to mitigate the Multi User Interference in MIMO based MC-CDM system named as Fuzzy Logic empowered Adaptive Back Propagation Neural Network (FLeABPNN). In proposed FLeABPNN, the learning rate of the backpropagation neural network is updated using fuzzy logic instead of using a constant learning rate. This improves the performance of the MIMO based MC-CDMA system as compared to the conventional LMS $[28,29]$ \& GA based suboptimum receiver $[16,33]$ in terms of Convergence rate \& Minimum Mean Square Error. Computational complexity is another challenging issue in modern communication [38-42]. The proposed FLeABPNN solution gives attractive results with low computational complexity.

The system model is described in Section [2]. The proposed FLeABPNN based MC-CDMA receiver model is described in Section [3]. Section [4] describes the 
simulation results and discussion. Finally, the research work is concluded in Section [5] \& Future work suggested in Section [6].

\section{System Model}

In this article, the MC-CDMA system used with Alamouti's STBC having two transmit and one receiver antenna. The number of both antennas can be increased as per the requirements. In the MC-CDMA receiver side signal received from the transmitter. After receiving the signal, a cyclic prefix is removed which adds at the transmitter end. Then taking the M-point Fast Fourier Transforms (FFT) after converting the data into serial to parallel. Finally, the received signal vector in the frequency domain is written as $[16,28,29,33]$ :

$$
\begin{array}{r}
r(2 i-1)=\sum_{u=1}^{U}\left\{\mathbf{f r}_{u, 1} \mathbf{S c}_{u, 1} e_{\mathbf{k}}(2 i-1)\right\}+\mathbf{f r}_{u, 2} \mathbf{S c}_{u, 2} e_{\mathbf{k}}(i)+\dot{\mathrm{z}}(2 i-1), \\
r(2 i)=\sum_{u=1}^{U}\left\{-\mathbf{f r}_{u, 1} \mathbf{S} \mathbf{c}_{u, 1} e_{k}^{*}(2 i)+\mathbf{f r}_{u, 2} \mathbf{S} \mathbf{c}_{u, 2} e_{k}^{*}(2 i-1)\right\}+\dot{\mathrm{z}}(2 i),
\end{array}
$$

where $U, \dot{z}$, fr \& Sc are representing the total number of users, complex Additive White Gaussian Noise (AWGN) with zero mean, frequency-domain channel response and spreading code respectively [33].

$$
\begin{aligned}
\Upsilon\left(w_{1}, w_{2}\right) & =E\left[\left|\mathbf{W}^{H} r(i)-e_{1}(i)\right|^{2}\right]= \\
& =E\left[\left|\mathbf{w}_{1}^{H} r(i)-e_{1}(i-1)\right|^{2}\right]+E\left[\left|\mathbf{w}_{2}^{H} r(i)-e_{1}(i)\right|^{2}\right]= \\
& =\Upsilon_{1}\left(\mathbf{w}_{\mathbf{1}}\right)+\Upsilon_{2}\left(\mathbf{w}_{2}\right)
\end{aligned}
$$

In the Mean Square Error (MSE) based receiver for MC-CDMA system with Alamouti's STBC achieved by using the following problem [16, 28, 29, 33].

$$
\left[\mathbf{w}_{1, \mathrm{opt}}, \mathbf{w}_{2, \mathrm{opt}}\right]=\arg \left\{\min _{\mathbf{w} 1} \Upsilon_{1}\left(\mathbf{w}_{1}\right)+\min _{\mathbf{w} 2} \Upsilon_{2}\left(\mathbf{w}_{2}\right)\right\}
$$

In [17-19] the relationship of weight vector is fulfilled as $[16,28,29,33]$ :

$$
\mathbf{w}_{1,2}=\mathbf{w}_{2,3}^{*}, \mathbf{w}_{1,4}=-\mathbf{w}_{1,1}^{*}
$$

The rate of convergence is increased by updating the weight of vectors. The improved MMSE based cost function can be written as [16, 28, 29,33]:

$$
\Upsilon=\Upsilon_{N 1}\left(\mathbf{w}_{d}, \mathbf{w}_{e}\right)+\Upsilon_{N 2}\left(\mathbf{w}_{d}, \mathbf{w}_{e}\right)
$$

where

$$
\Upsilon_{N 1}\left(\mathbf{w}_{d}, \mathbf{w}_{e}\right)=E\left[\left|\mathbf{w}_{d}^{H} r(i-1)+\mathbf{w}_{e}^{T} r^{*}(2 i)-e_{1}(i-1)\right|^{2}\right]
$$

and

$$
\Upsilon_{N 2}\left(\mathbf{w}_{d}, \mathbf{w}_{e}\right)=E\left[\left|\mathbf{w}_{e}^{H} r(i-1)+\mathbf{w}_{d}^{T} r^{*}(2 i)-e_{1}(i)\right|^{2}\right]
$$




\section{Proposed fuzzy logic empowered adaptive back propagation neural network (FLeABPNN) based MUD}

Fig. 1 shows the proposed FLeABPNN based MC-CDMA receiver model in which the received signal is down-converted for the u-th user. The cyclic prefixes are removed in the first step after that signal is transferred from serial to parallel. After taking the FFT proposed FLeABPNN is used to optimize the weights of the receiver.

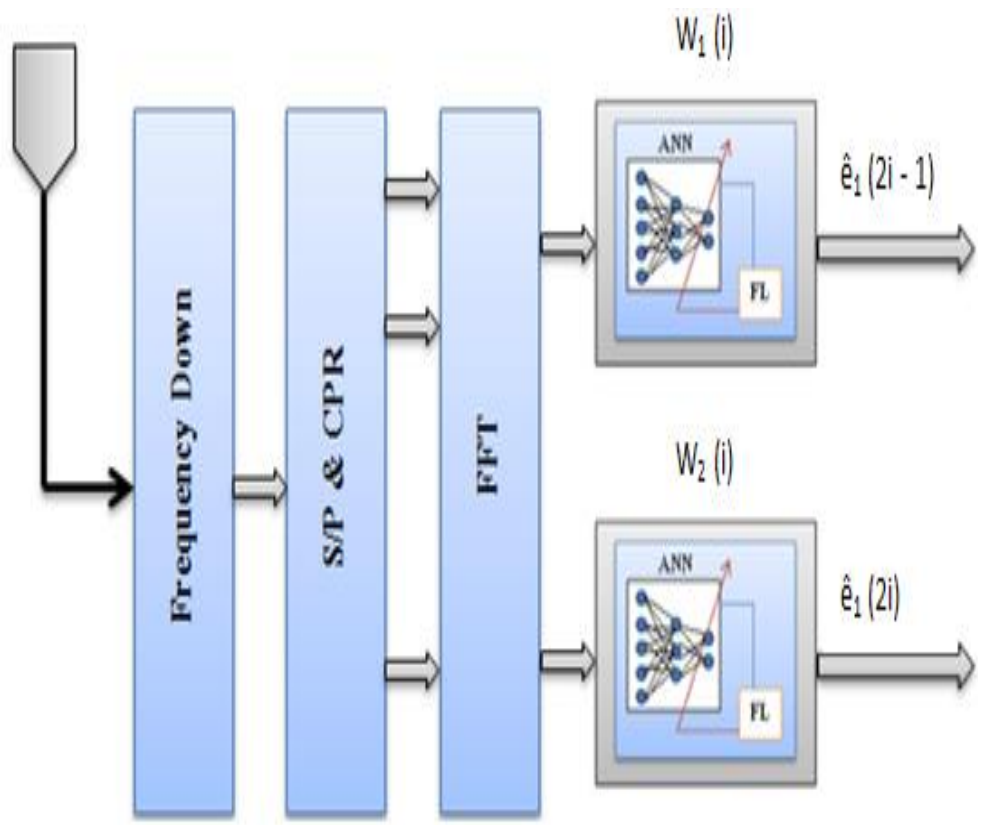

Fig. 1 Proposed FLeABPNN based receiver system.

Proposed FLeABPNN has used a total of three layers like input, hidden and an output layer. Different steps are involved in the algorithm of backpropagation which includes, Initialization of weight, Feedforward, Back Propagation of error and updating of weight and bias as shown in Tab. I. Every neuron present in the hidden layer has a Sigmoid activation function. Proposed FLeABPNN based MC-CDMA system can be written as

$$
\begin{gathered}
\psi_{j}=b_{1}+\sum_{i=1}^{m}\left(\omega_{i j} \times \mathbf{r}_{i}\right), \\
\varphi_{j}=\frac{1}{1+\mathbf{e}^{-\psi_{j}}}, \text { where } j=1,2,3 \ldots n .
\end{gathered}
$$




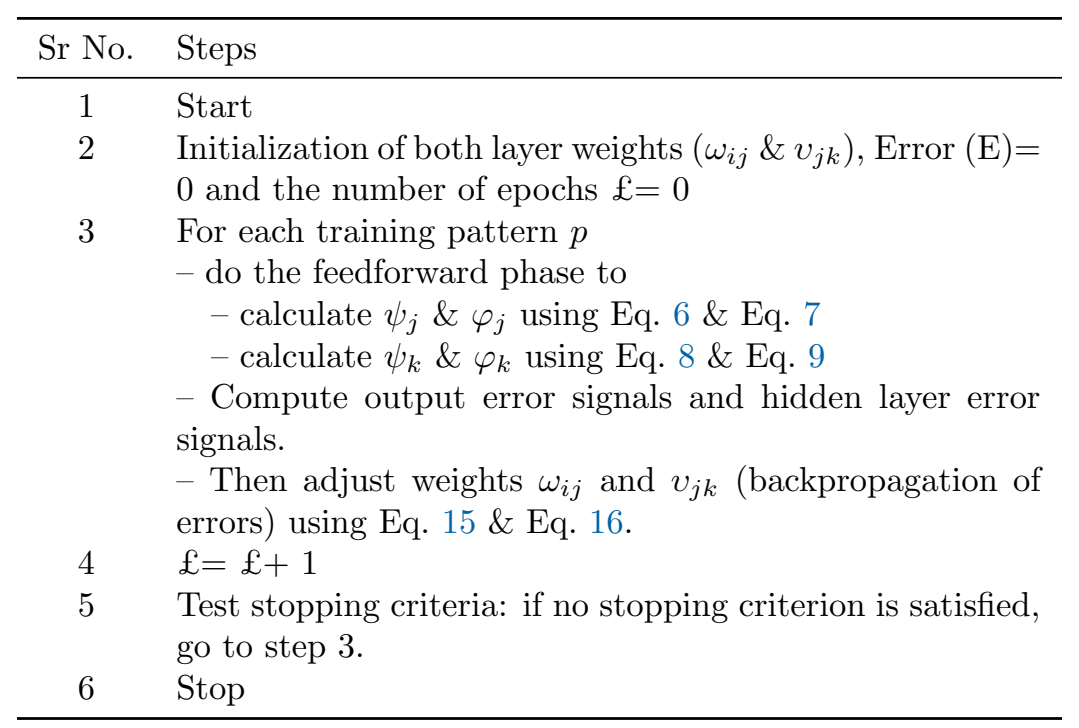

Tab. I Pseudocode of proposed FLeABPNN based $M C$-CDMA receiver.

Input taken from the output layer is

$$
\psi_{k}=b_{2}+\sum_{j=1}^{n}\left(v_{j k} \times \varphi_{j}\right)
$$

Output layer activation function is given below.

$$
\begin{gathered}
\varphi_{k}=\frac{1}{1+\mathrm{e}^{-\psi_{k}}} \quad \text { where } k=1,2,3 \ldots r \\
E=\frac{1}{2} \sum_{k}\left(\tau_{k}-\varphi_{k}\right)^{2}
\end{gathered}
$$

Above equation represents back propagation error where, $\tau_{k} \&$ out $_{k}$ represent the desired output and estimated output.

Then rate of change in weight for the output, the layer is written as.

$$
\begin{array}{r}
\Delta W \propto-\frac{\partial E}{\partial W} \\
\Delta v_{j, k}=-\epsilon \frac{\partial E}{\partial \nu_{j, k}}
\end{array}
$$

After applying the Chain rule method Eq. 11 can be written as

$$
\Delta v_{j, k}=-\epsilon \frac{\partial E}{\partial \varphi_{k}} \times \frac{\partial \varphi_{k}}{\partial \psi_{k}} \times \frac{\partial \psi_{k}}{\partial \nu_{j, k}}
$$


After substituting the values in Eq. 12 the value of weight changed can be obtained as shown in Eq. 13.

$$
\begin{array}{r}
\Delta v_{j, k}=\epsilon\left(\tau_{k}-\varphi_{k}\right) \times \varphi_{k}\left(1-\varphi_{k}\right) \times\left(\varphi_{j}\right) \\
\Delta v_{j, k}=\epsilon \xi_{k} \varphi_{j},
\end{array}
$$

where

$$
\xi_{k}=\left(\tau_{k}-\varphi_{k}\right) \times \varphi_{k}\left(1-\varphi_{k}\right) .
$$

Apply chain rule for the updating weights between input and hidden layers

$$
\begin{aligned}
& \Delta \omega_{i, j} \propto-\left[\sum_{k} \frac{\partial \mathbf{E}}{\partial \varphi_{k}} \times \frac{\partial \varphi_{k}}{\partial \psi_{k}} \times \frac{\partial \psi_{k}}{\partial \varphi_{j}}\right] \times \frac{\partial \varphi_{j}}{\partial \psi_{j}} \times \frac{\partial \psi_{j}}{\partial \omega_{i, j}} \\
& \Delta \omega_{i, j}=-\epsilon\left[\sum_{k} \frac{\partial \mathbf{E}}{\partial \varphi_{k}} \times \frac{\partial \varphi_{k}}{\partial \psi_{k}} \times \frac{\partial \psi_{k}}{\partial \varphi_{j}}\right] \times \frac{\partial \varphi_{j}}{\partial \psi_{j}} \times \frac{\partial \psi_{j}}{\partial \omega_{i, j}}
\end{aligned}
$$

In the above equation, $\epsilon$ represents the constant,

$$
\begin{aligned}
& \Delta \omega_{i, j}=\epsilon\left[\sum_{k}\left(\tau_{k}-\varphi_{k}\right) \times \varphi_{k}\left(1-\varphi_{k}\right) \times\left(\nu_{j, k}\right)\right] \times \varphi_{k}\left(1-\varphi_{k}\right) \times \alpha_{i} \\
& \Delta \omega_{i, j}=\epsilon\left[\sum_{k}\left(\tau_{k}-\varphi_{k}\right) \times \varphi_{k}\left(1-\varphi_{k}\right) \times\left(\nu_{j, k}\right)\right] \times \varphi_{j}\left(1-\varphi_{j}\right) \times \alpha_{i} \\
& \Delta \omega_{i, j}=\epsilon\left[\sum_{k} \xi_{k}\left(\nu_{j, k}\right)\right] \times \varphi_{j}\left(1-\varphi_{j}\right) \times \alpha_{i} .
\end{aligned}
$$

After simplification above equation can be written as

$$
\Delta \omega_{i, j}=\epsilon \xi_{j} \alpha_{i}
$$

where

$$
\begin{gathered}
\xi_{j}=\left[\sum_{k} \xi_{k}\left(\nu_{j, k}\right)\right] \times \varphi_{j}\left(1-\varphi_{j}\right) \\
\nu_{j, k}^{+}=\nu_{j, k}+\lambda_{F} \Delta v_{j, k}
\end{gathered}
$$

The Eq. 15 is used for updating the weights between output \& hidden layers.

$$
\omega_{i, j}^{+}=\omega_{i, j}+\lambda_{F} \Delta \omega_{i, j}
$$

And the above equation is used for updating the weights between the input layer and the hidden layer.

In the above equations $\lambda_{F}$ represents the learning rate of ABPNN. Convergence of ABPNN depends upon the careful selection of $\lambda_{F}$. In this article $\lambda_{F}$ is updated using fuzzy logic as shown in given bellow

$$
\lambda_{F}=F L C\left(e_{s}, \Delta e_{s}\right),
$$




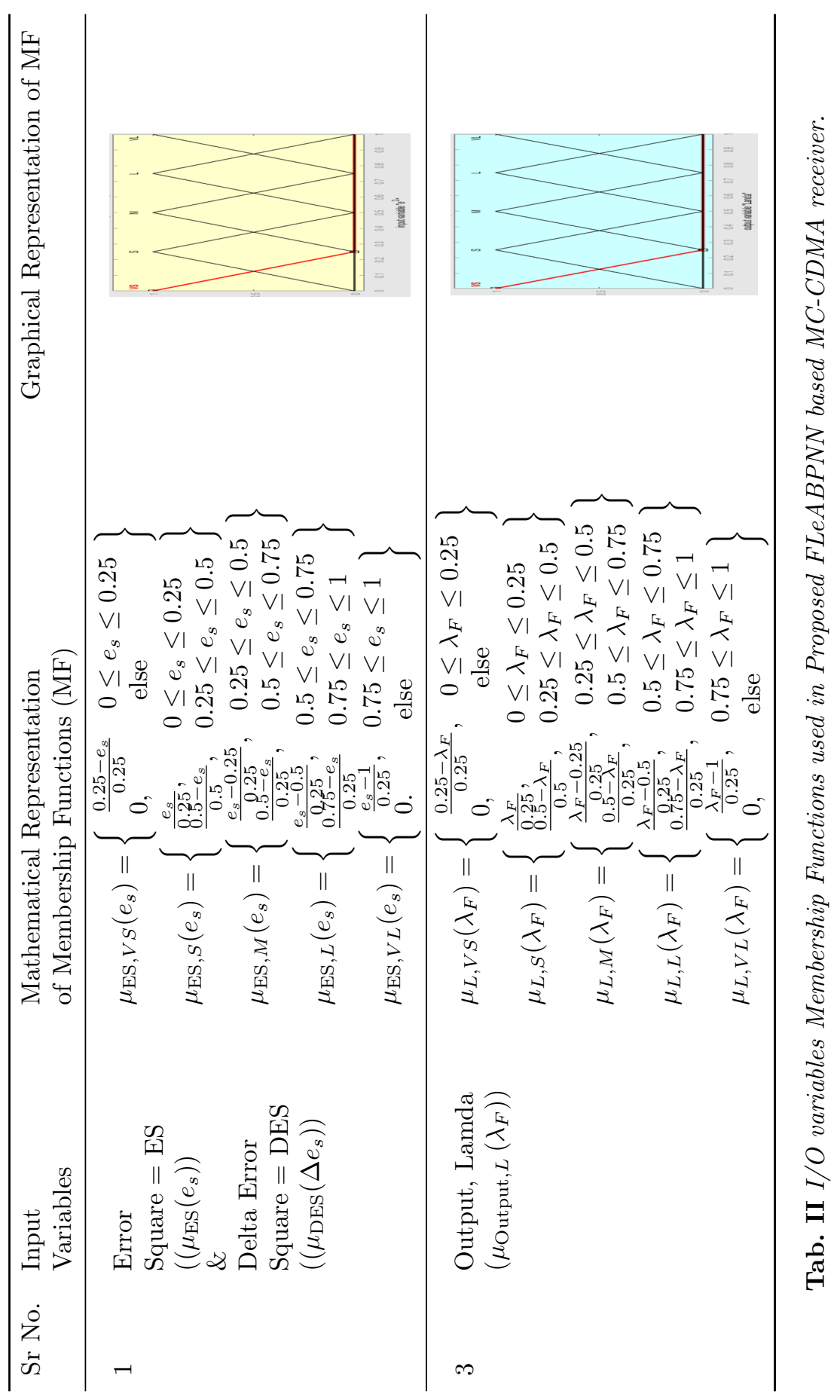


where $e_{s}$ and $\Delta e_{s}$ are mean square error and normalized mean square error of the Proposed FLeABPNN system.

$$
\Delta e_{s}=e_{s}(t)-e_{s}(t-1)
$$

Mathematically and graphically Input / Output variable membership functions used in the Proposed FLeABPNN based system are shown in Tab. II.

There are four main parts in the fuzzy systems named as fuzzy prepositions, lookup table, inference engine and De- Fuzzifier.

The fuzzy preposition t-norm function is written as

$$
t: e_{s}, \Delta e_{s} \rightarrow \lambda_{F}
$$

the above equation can also be written in terms of fuzzy sets

$$
\begin{aligned}
t:[0,1] & \times[0,1] \rightarrow[0,1] \\
{\left[\left(\mu_{\mathrm{ES}}\left(e_{s}\right),\left(\mu_{\mathrm{DES}}\left(\Delta e_{s}\right)\right]\right.\right.} & =\min \left[\left(\mu_{E S}\left(e_{s}\right),\left(\mu_{\mathrm{DES}}\left(\Delta e_{s}\right)\right],\right.\right. \\
& =\mu_{\mathrm{ES}, \mathrm{DES}}\left(e_{s} \Delta e_{s}\right)
\end{aligned}
$$

The proposed FLeABPNN based MC-CDMA receiver lookup table consists of 10 I/O rules from 25 rules as shown in Tab. III.

\begin{tabular}{clll}
\hline Rules & Error Square (es) & Delta Error Square $(\Delta \mathrm{es})$ & Lambda $(\lambda)$ \\
\hline 1 & VS (Very Small) & VS (Very Small) & VS (Very Small) \\
2 & VS (Very Small) & S (Small) & VS (Very Small) \\
3 & VS (Very Small) & M (Medium) & S (Small) \\
4 & VS (Very Small) & L (Large) & M (Medium) \\
5 & VS (Very Small) & VL (Very Large) & M (Medium) \\
6 & S (Small) & VS (Very Small) & VS (Very Small) \\
7 & S (Small) & S (Small) & S (Small) \\
8 & S (Small) & M (Medium) & S (Small) \\
9 & S (Small) & L (Large) & M (Medium) \\
10 & S (Small) & VL (Very Large) & M (Medium) \\
\hline
\end{tabular}

Tab. III Lookup table for proposed FLeABPNN based MC-CDMA receiver

In the fuzzy system membership function, IF-THEN rules are used as conditional statements. The important parts of fuzzy inference systems contain rules surface, rules viewer, etc. Proposed FLeABPNN based MC-CDMA receiver contains 25 rules denoted by $\mathrm{Rv}^{\eta}$, where $1 \leq \eta \leq 25$.

$-\mathrm{Rv}^{1}=\mathrm{IF}$ error square is very Small AND delta error square is very Small THEN lambda is very Small

$-\mathrm{Rv}^{2}=\mathrm{IF}$ error square is very Small AND delta error square is Small THEN lambda is very Small 
- $R v^{25}=\mathrm{IF}$ error square is very large AND delta error square is very large THEN lambda is very large

Proposed FLeABPNN based MC-CDMA receiver inference engine $R v^{\eta}$ can be written as

$$
R v^{\eta}=\mathrm{ES}^{\eta} \times \mathrm{DES}^{\eta} \rightarrow \lambda_{F}^{\eta}
$$

The above equation can be written as

$$
\left.\mu_{\mathrm{ES} \cap \mathrm{DES}}\left(e_{s}, \Delta e_{s}\right)=\mu_{\mathrm{ES}}\left(e_{s}\right) \cap \mu_{\mathrm{DES}}\left(\Delta e_{s}\right)\right]
$$

The rules are interpreted as a single fuzzy relation defined by

$$
R_{25}=\bigcup_{\eta=1}^{25} R v^{\eta}
$$

Assume two fuzzy sets named as $\aleph$ and $\emptyset$ also input and output of fuzzy inference engine individually. For $\mathrm{R}_{25}$ the output of Fuzzy Inference Engine is written as

$$
\begin{aligned}
& \mu_{\text {Very SlownSlownMedium } \cap \text { LargenVery Large }}(\emptyset) \\
= & \sup _{\aleph \in(\mathrm{ES}, \mathrm{DES})} t\left[\mu_{\aleph}\left(e_{s}, \Delta e_{s}\right), \mu_{R_{25}}\left(e_{s}, \Delta e_{s}, \lambda_{F}\right)\right]
\end{aligned}
$$

The Proposed FLeABPNN based MC-CDMA receiver Product Inference Engine (PIE) can be written as

$$
\begin{aligned}
& \mu_{\xi} \text { Lambda }= \\
= & \max _{1 \leq \eta \leq 25}\left[\sup _{\aleph \in(\mathrm{ES}, \mathrm{DES})}\left(\prod_{j=1}^{25}\left(\mu_{\mathrm{ES}, \mathrm{DES}}(\mathrm{ES}, \mathrm{DES}), \mu_{A_{1 \aleph} A_{2 \aleph}}\left(a_{1}, a_{2}\right)\right)\right)\right]
\end{aligned}
$$

Center of Gravity (CoG) DE fuzzifier is used in the Proposed FLeABPNN based MC-CDMA receiver. The CoG DE fuzzifier determines the $\mathrm{CE}$ as the center of the area as shown in Eq. 26 covered by the membership function of $\emptyset$, that is,

$$
\mathrm{CE}=\frac{\int \xi \mu_{\xi}(\xi) \mathrm{d} \xi}{\int \mu_{\xi}(\xi) \mathrm{d} \xi}
$$

Fig. 2 shows the ruled surface of the Proposed FLeABPNN based MC-CDMA receiver. It is shown that if $e_{s}$ and $\Delta e_{s}$ are very small then $\lambda_{F}$ is very small. Similarly, if $e_{s}$ and $\Delta e_{s}$ are very large then $\lambda_{F}$ is very large.

Fig. 3 shown that if $e_{s}$ is very small and $\Delta e_{s}$ is small then change in learning rate $\lambda_{F}$ of the proposed model is very small.

Fig. 4 shown that if $e_{s}$ is very small and $\Delta e_{s}$ is medium then learning rate $\lambda_{F}$ of the proposed model is small. Similarly, Fig. 5 shows that if $e_{s}$ is small and $\Delta e_{s}$ is large then learning rate $\lambda_{F}$ of the proposed model is medium.

Fig. 6 shows that the proposed model learning rate $\lambda_{F}$ is large if $e_{s}$ is medium and $\Delta e_{s}$ is very high.

Similarly, Fig. 7 shows that if $e_{s}$ is large and $\Delta e_{s}$ is very large then proposed model learning rate $\lambda_{F}$ is also very large. 
Abbas S. et al.: Multi user detection using fuzzy logic empowered...

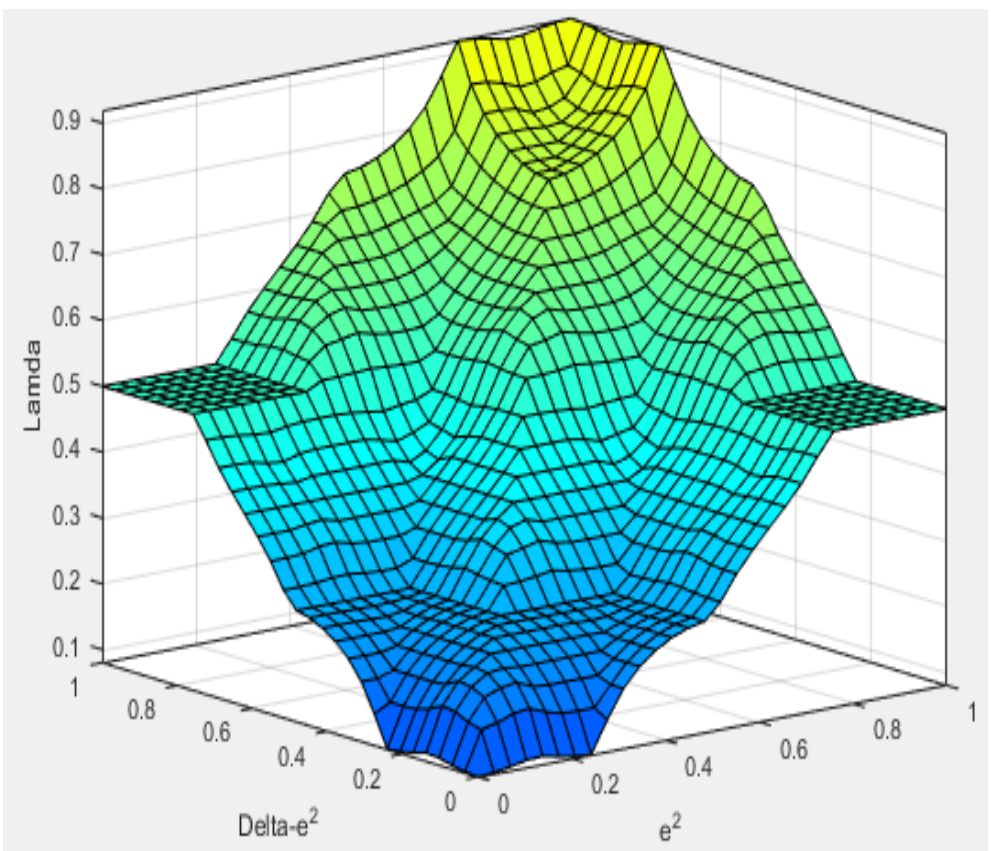

Fig. 2 Proposed FLeABPNN based $M C$-CDMA receiver ruled surface.

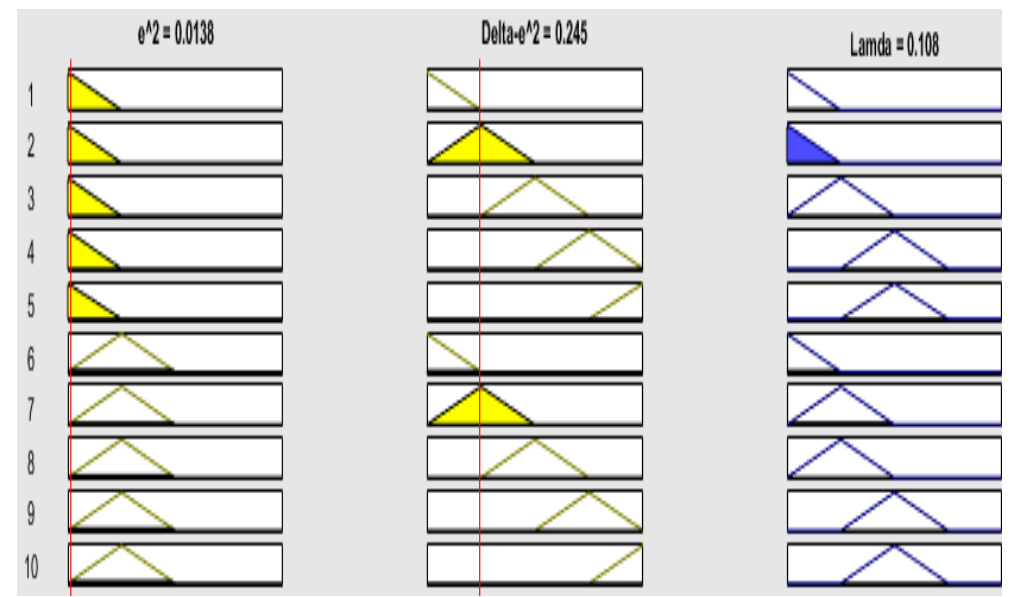

Fig. 3 Proposed FLeABPNN based receiver Look up diagram for $\lambda_{F}$ is very small.

\section{Results Analysis and Findings}

MATLAB 2018 is used for simulation purposes. The MC-CDMA system is employed with $\mathrm{A}=32$ subcarriers, which is equal to the dimension of spreading code. The subcarrier spacing is $312 \mathrm{~Hz}$ and the time duration at the subcarriers is $0.32 \mathrm{~ms}$. The spreading code real and imaginary parts are selected from $1 / \sqrt{ } 2$ and 


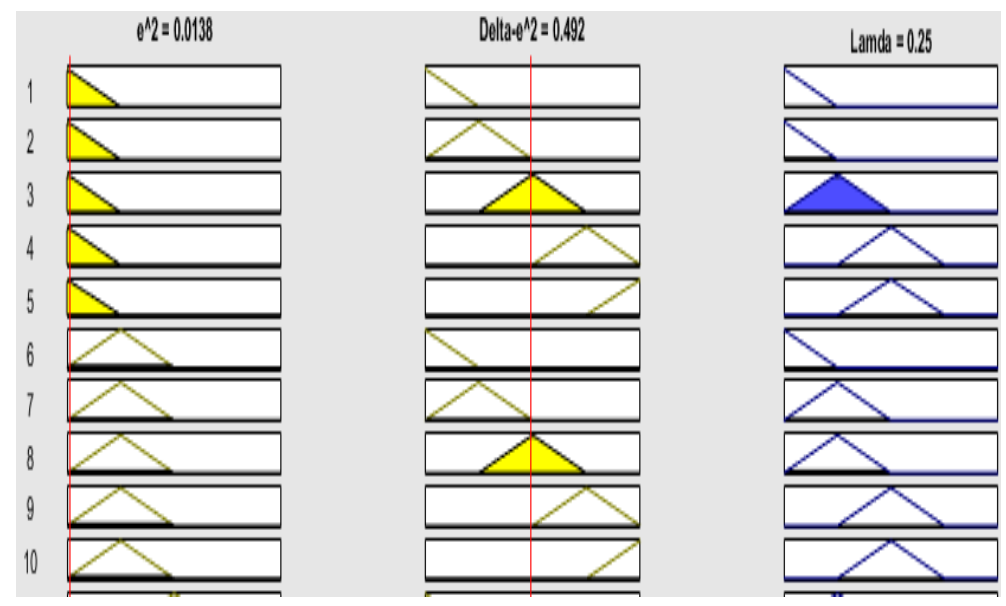

Fig. 4 Proposed FLeABPNN based receiver Look up diagram for $\lambda_{F}$ is small.

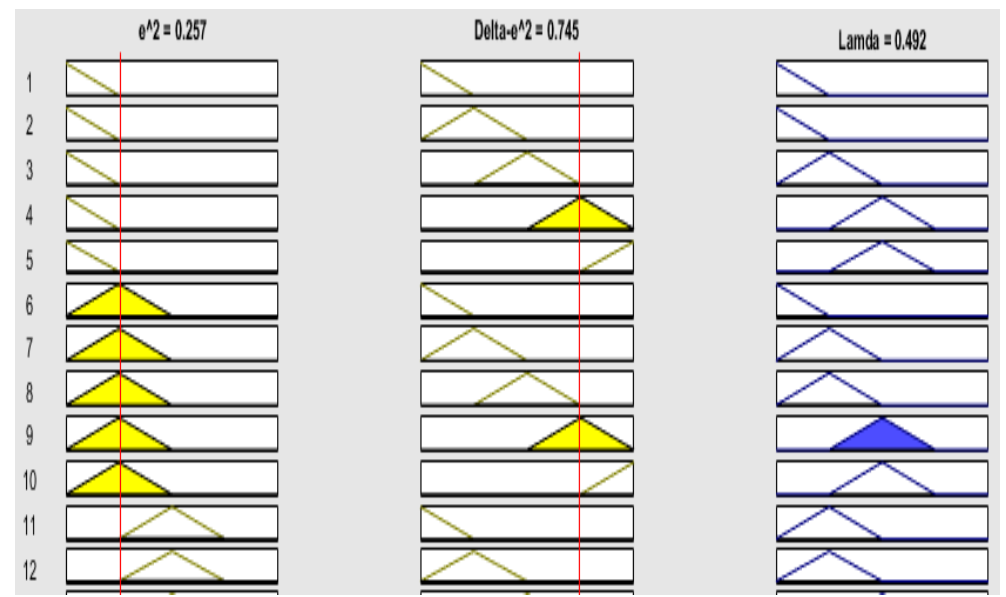

Fig. 5 Proposed FLeABPNN based receiver Look up diagram for $\lambda_{F}$ is medium.

$-1 / \sqrt{ } 2$ independently at random for each user. The Rayleigh flat fading channel is implemented using four paths. The fading gains are generated by using a complex Gaussian distribution, which are normalized such that the average energy of the channel is unity. The channel coefficients along spreading codes are fixed for whole observation.

The Figs. 8, 9, 10 and 11 signifies FLeABPNN based MC-CDMA receiver performance with respect to Minimum Mean Square Error (MMSE), convergence rate and complexity factor.

Fig. 8 shows the NoC vs MMSE with a fixed number of Hidden layers for both enhanced and a basic cost function with SNR is $25 \mathrm{~dB}$. The 1-st, 2-nd, 3-rd \& 4-th topmost curves show the performance in terms of MMSE of conventional (LMS) and accelerated GA based scheme without relationship as well as with relationship. 
Abbas S. et al.: Multi user detection using fuzzy logic empowered...

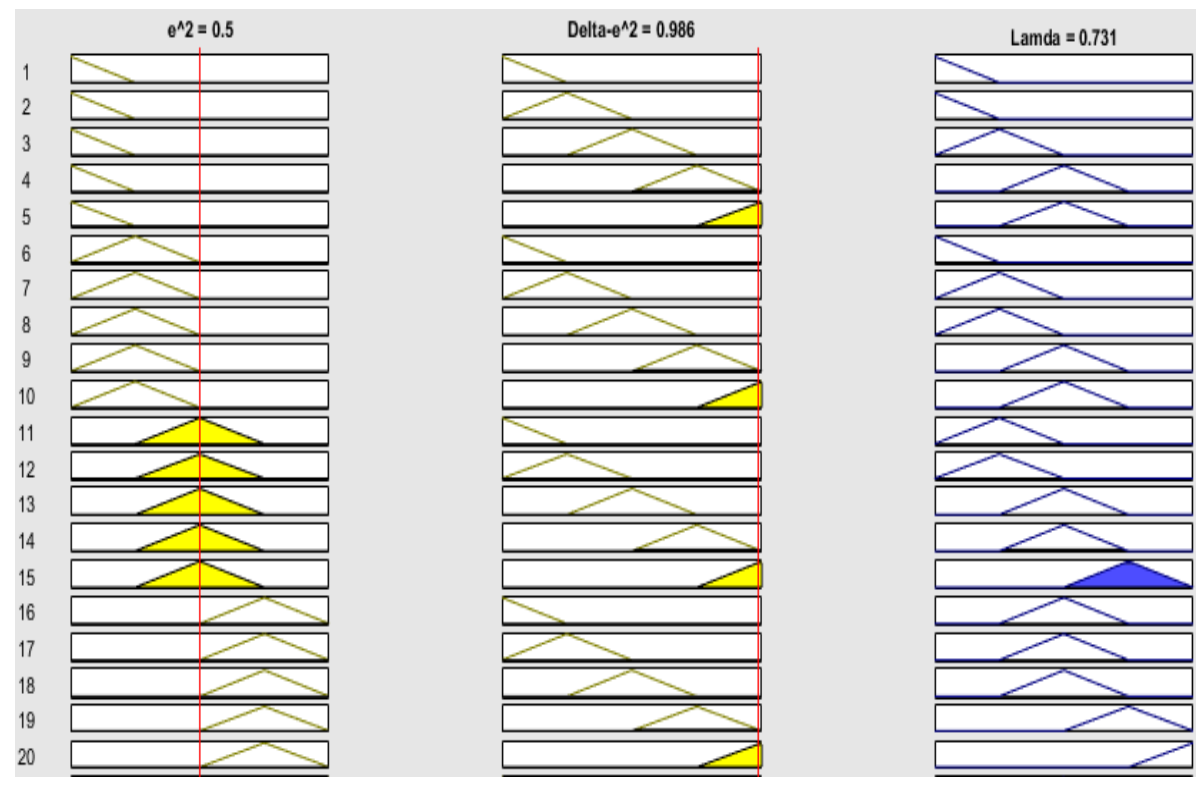

Fig. 6 Proposed FLeABPNN based receiver Look up diagram for $\lambda_{F}$ is large.

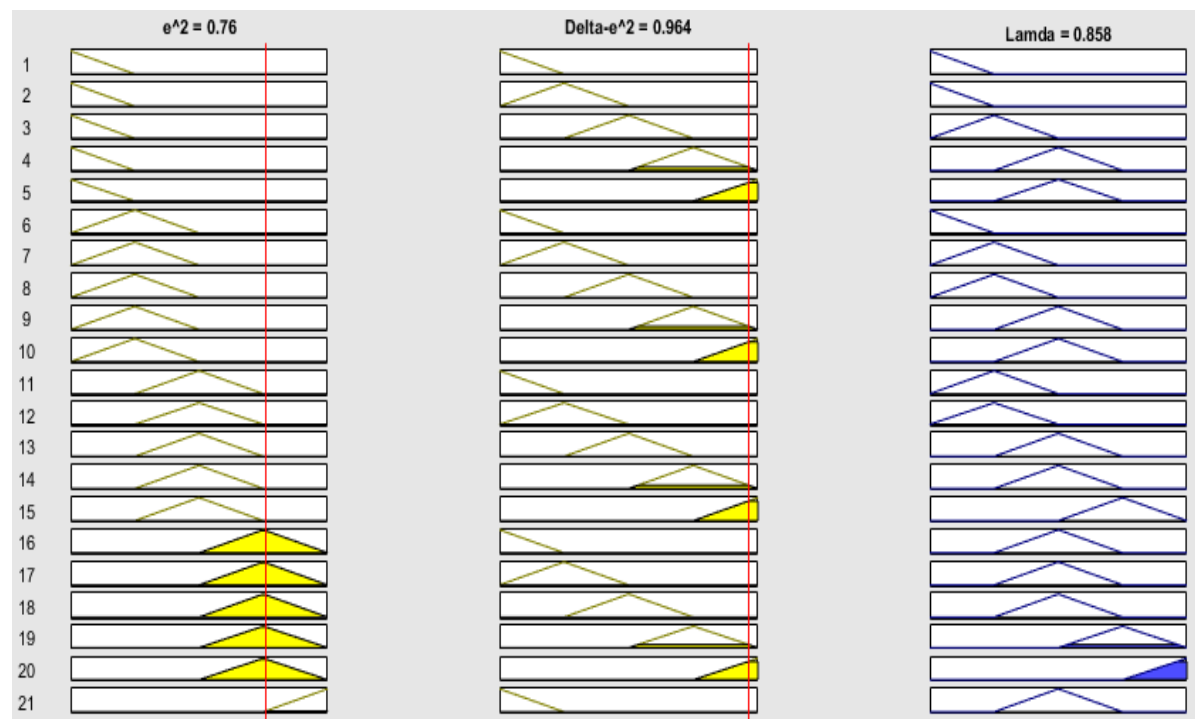

Fig. 7 Proposed FLeABPNN based receiver Look up diagram for $\lambda_{F}$ is very large.

It demonstrates that the LMS scheme without relationship \& with relationship converges at 350-th, 250-th iteration to reach the same MMSE of 0.03 respectively. It very well may be seen that the accelerated GA based receiver without relationship \& with relationship converges at 166-th, 90-th epoch respectively. The 2-nd last curve represents the proposed FLeABPNN based MC-CDMA receiver without a 


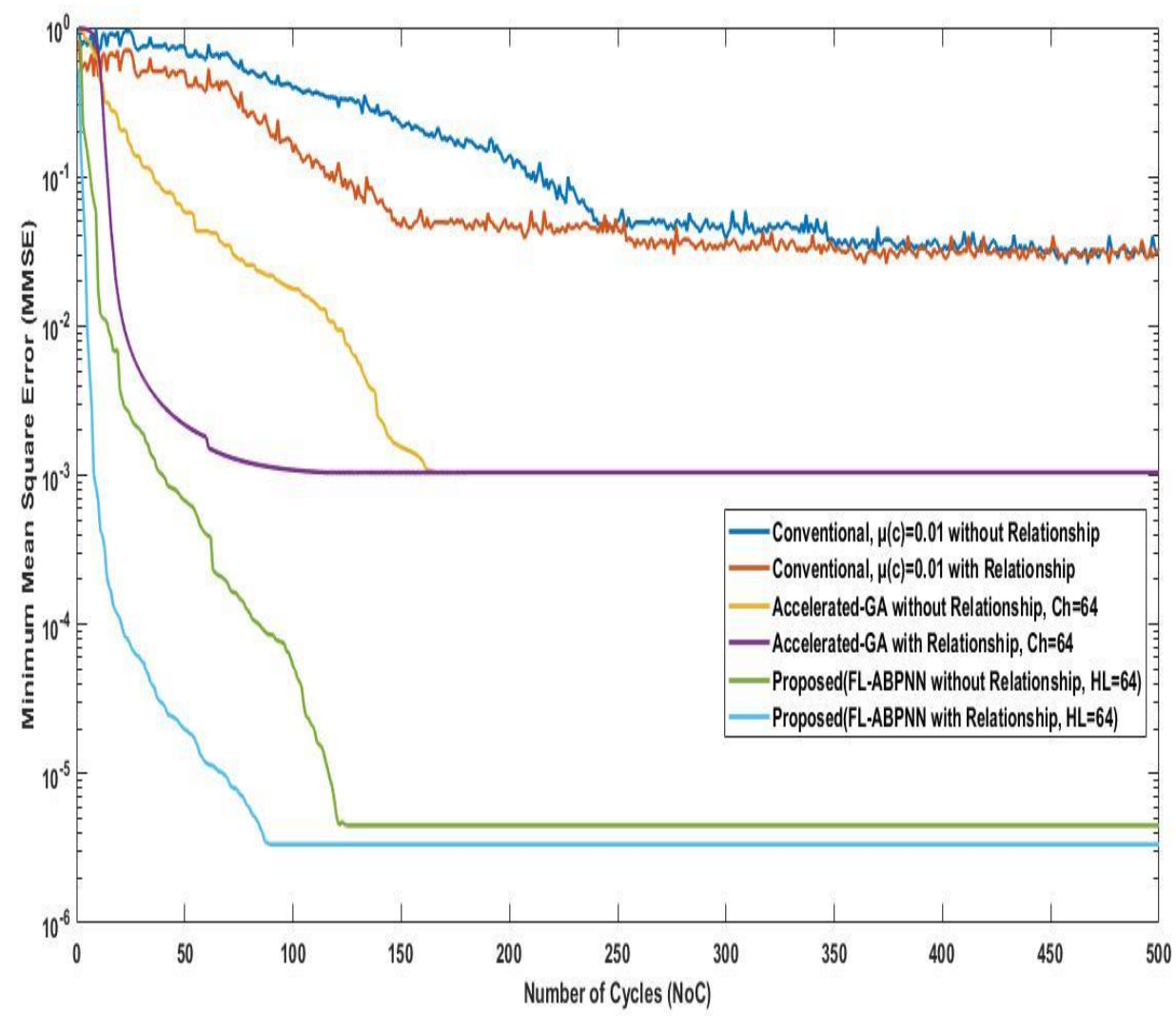

Fig. 8 Minimum mean square error vs number of cycles (fixed hidden layers $=10$ ).

relationship. The bottom curve represents the proposed FLeABPNN based MCCDMA receiver with the relationship. It very well may be seen the FLeABPNN receiver with relationship, converges at the 87-th cycle to achieve the MMSE of $3.4 \times 10^{-06}$. The FLeABPNN based MC-CDMA receiver without the relationship, converges at approximately 125-th cycle to achieve the MMSE of $4.5 \times 10^{-06}$. Hence, it very well may be said that FLeABPNN based MC-CDMA receiver with relationship converges faster, and lower MMSE than the FLeABPNN based MCCDMA receiver without the relationship as well as conventional \& accelerated GA schemes.

Fig. 9 demonstrates a number of users Vs factor of the complexity of conventional, accelerated GA and proposed FLeABPNN based MC-CDMA receiver schemes with both variations. The GA and conventional scheme complexity factor are calculated by $\frac{2^{k}}{\text { generations } \times \text { chromosomes }}$ and $\mathrm{k}(\mathrm{B}-1)(\mathrm{B}+1)$ respectively [20]. And the complexity factor of FLeABPNN based MC-CDMA receiver scheme is calculated by

$$
\frac{2^{k}}{\text { Input Layer Neurons } \times \text { Output Layer Neurons } \times \text { Hidden Layer Neurons }}
$$


Abbas S. et al.: Multi user detection using fuzzy logic empowered...

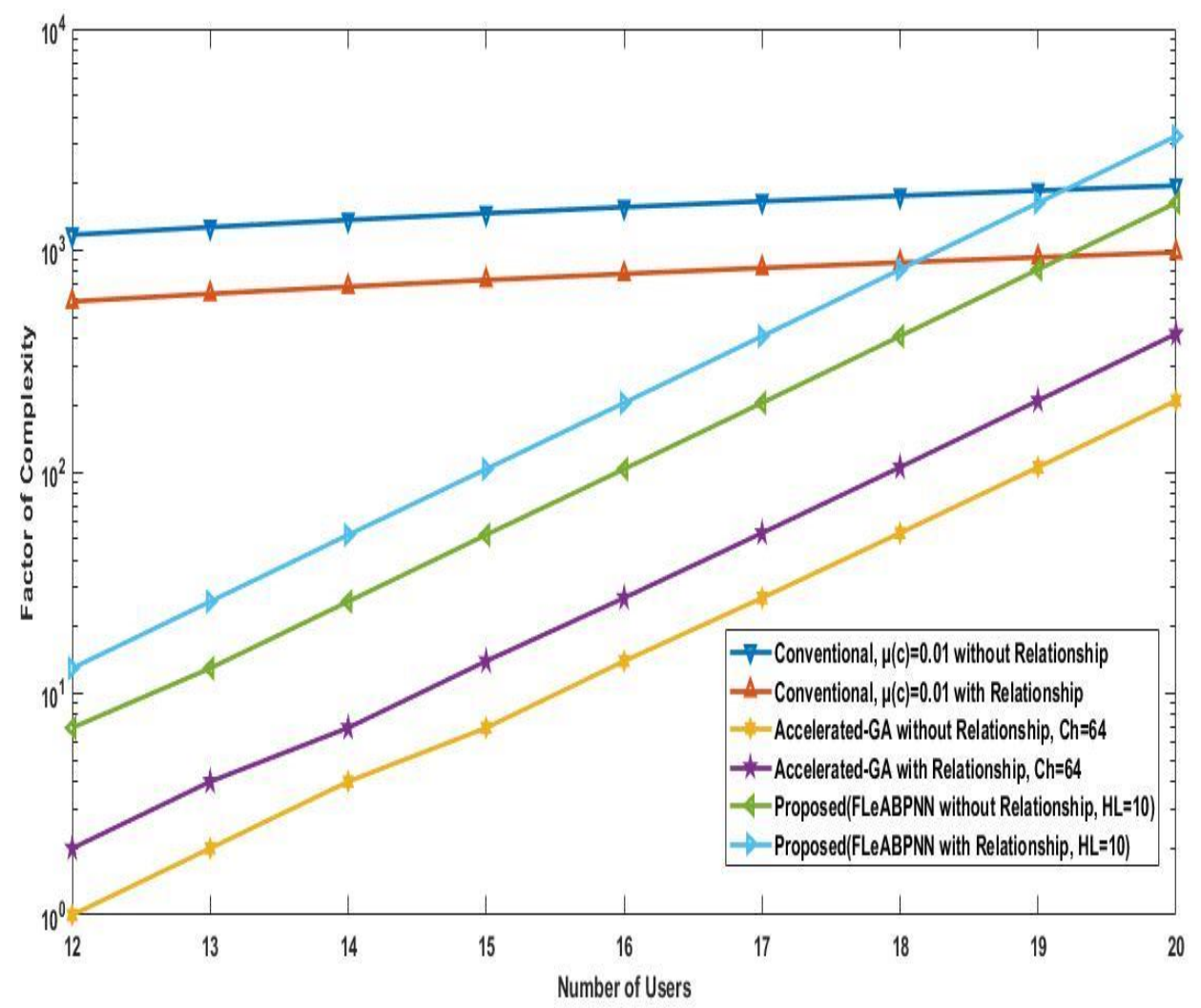

Fig. 9 Complexity factor vs no of users with a fixed number of hidden layers.

It very well may be seen in Fig. 9; the complexity factor has elevated up to $18 \mathrm{~dB}$ SNR. The LMS has the maximum complexity as compared to propose FLeABPNN based MC-CDMA receiver variations. The proposed FLeABPNN based MC-CDMA receiver with the maximum number of hidden layers $(\mathrm{HL}=10)$ has the least complexity as compared to the conventional scheme. But Proposed FLeABPNN based MC-CDMA receiver complexity is higher as compared to accelerated GA based receiver. However, it seems from the behavior of the graphs that proposed FLeABPNN based MC-CDMA receiver with weight relationship has less complexity as compared to FLeABPNN based MC-CDMA receiver without weight relationship \& conventional LMS based on MC-CDMA system.

Fig. 10 demonstrates the number of users vs the complexity factor for attaining the different bit error rate \& a number of hidden layers. The conventional LMS is limited to $10^{-1.8} \mathrm{BER}$, accelerated GA is restricted to $10^{-3} \mathrm{BER}$, whereas the proposed FLeABPNN based MC-CDMA receiver is limited to 3 different BER's starting from $10^{-3}$ to $10^{-5.5}$. It is seen from the above figure that the proposed FLeABPNN based MC-CDMA receiver reaches low BER with fewer complications as compared to conventional LMS scheme up to 17 number of users, but, proposed FLeABPNN based MC-CDMA receiver attains low BER as compared to acceler- 
ated GA at the cost of complexity. It also observed that the complexity factor is also increased with the increase in a number of hidden layers.

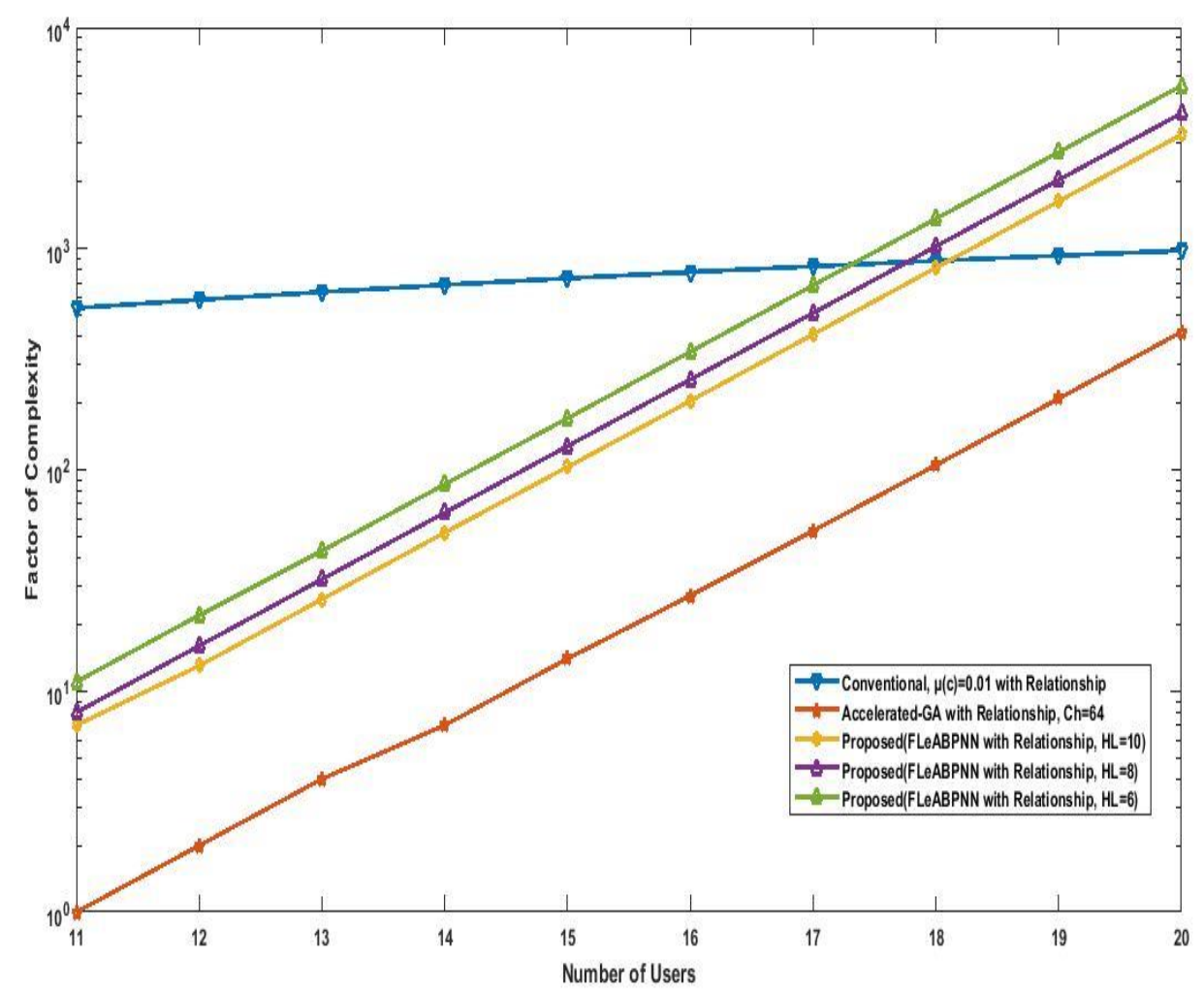

Fig. 10 Factor of complexity vs number of user's with different number of hidden layers.

Fig. 11 shows the number of user's vs Time Complexity (TC) for achieving the different bit error rates with the relationship. The arc illustrations from topmost to bottommost are:

- It is observed that the conventional scheme needs approximately $10^{4}$ epochs to attain the 0.001 BER approximately.

- The accelerated GA with relationship limited at a $10^{-3}$ bit error rate with the number of chromosomes $=64$. It might be seen that it needs $10^{5.9}$ epochs for 20 users with the end goal to accomplish $\mathrm{BER}=10^{-3}$.

- The 2-nd arc is for proposed FLeABPNN based MC-CDMA receiver with relationship restricted at $10^{-3}$ bit error rate and the number of hidden layers $=6$. It very well may be seen that it required $10^{4.7}$ epochs for 20 users in order to attain the $\mathrm{BER}=10^{-3}$.

- The 3-rd arc is for proposed FLeABPNN based MC-CDMA receiver with relationship restricted at $10^{-3}$ bit error rate and the number of hidden layers 
Abbas S. et al.: Multi user detection using fuzzy logic empowered...

$=8$ It very well may be seen that it required $10^{4.8}$ epochs for 20 users in order to reach the $\mathrm{BER}=10^{-4.5}$.

- The 4-th arc is for proposed FLeABPNN based MC-CDMA receiver with relationship restricted at $10^{-3}$ bit error rate and a number of hidden layers $=10$. It very well may be seen that it required $10^{4.9}$ epochs for 20 users in order to reach the $\mathrm{BER}=10^{-5.5}$.

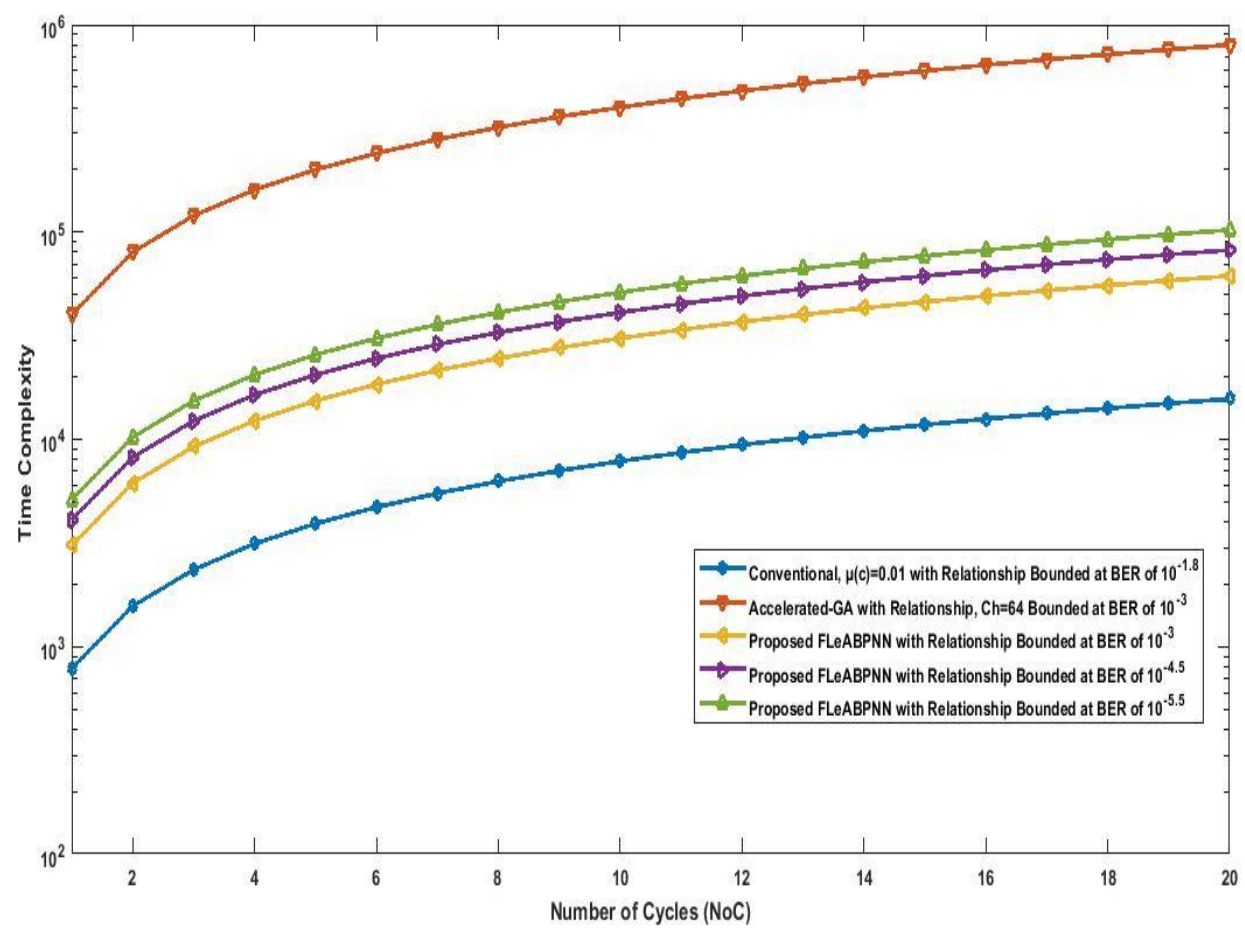

Fig. 11 Number of user's vs time complexity for achieving the different BER with relationship.

Hence, it very well may be said that the proposed FLeABPNN based MCCDMA receiver with a relationship requires fewer cycles to accomplish any estimation of bit error rate for any number of users $\mathrm{K}$ as compared to accelerated GA receiver with the relationship.

Fig. 12 shows the Signal to Noise Ratio (SNR) vs Bit Error Rate (BER) with a fixed number of Hidden layers for both enhanced and a basic cost function with NoC, which is 250 . The 1 st, 2 nd, 3 rd \& 4 rth topmost curves show the performance in terms of BER of conventional (LMS) and accelerated GA based scheme without relationship as well as with relationship. It demonstrates that LMS scheme without relationship \& with relationship gives $0.1202 \& 0.1002$ respectively at $20 \mathrm{~dB}$ SNR. It very well may be seen that the accelerated GA based receiver without relationship \& with relationship gives $5.15 \times 10^{-3} \& 1.9 \times 10^{-3}$, respectively at $20 \mathrm{~dB}$ SNR. 


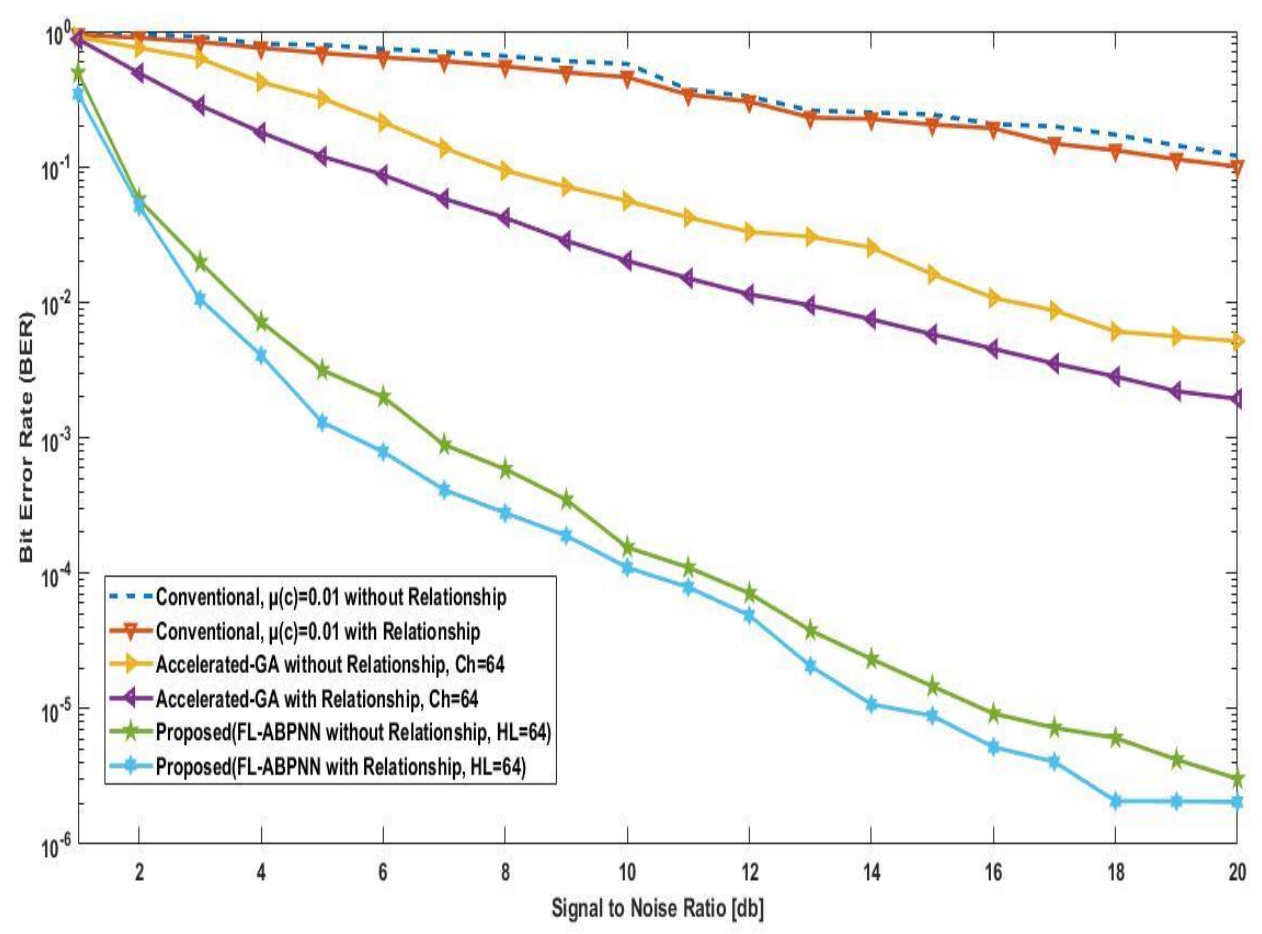

Fig. 12 SNR vs BER (fixed hidden layers $=10$ ).

The 2nd last curve represents the proposed FLeABPNN based MC-CDMA receiver without a relationship. The bottom curve represents the proposed FLeABPNN based MC-CDMA receiver with the relationship. At SNR $=20 \mathrm{~dB}$ it very well may be seen the FLeABPNN receiver without relationship \& with relationship achieve the BER of $3.04 \times 10^{-06} \& 2.05 \times 10^{-06}$, respectively. The FLeABPNN based MIMO-MC-CDMA receiver with a relationship gives more attractive BER as compared to others.

\section{Conclusions}

Modern contemporary technology world has given extensive rise to the number of users \& multimedia applications. Futuristic technological models are leading to high data demand and fast convergence rate networks. This article attempted a hybrid solution of fuzzy and neural network for MUD. The proposed FLeABPNN based MIMO-MC-CDMA receiver gives the $2.05 \times 10^{-06} \mathrm{BER} \&$ converges at the 87 -th cycle to achieve the MMSE of $3.4 \times 10^{-06}$ which are very attractive results as compared to previous published suboptimal based solutions like GA [33] \& LMS [28, 29]. It is also observed that the proposed FLeABPNN based MC-CDMA receiver with weight relation converges faster with low BER \& computational complexity as compared to without weight relationship-based solution. 
Abbas S. et al.: Multi user detection using fuzzy logic empowered...

\section{Future Research Directions}

The channel estimation is still not studied in this article; its mutual optimization along MUD is also another area of research. The mutation method of swarm algorithms can also be explored for further improvements. The model proposed in this article can be implemented in the field of medical diagnosis systems, channel equalization \& channel estimation for accuracy and efficiency. Other vast areas of medicine and pharmacology can also be tested under this model.

\section{References}

[1] PRASAD R., HARA S. An overview of multi-carrier CDMA. 4th International Symposium on Spread Spectrum Techniques and Applications; 1996, pp. 107-114. IEEE. doi: 10.1109/ isssta.1996.563752.

[2] HARA S., PRASAD R. Design and performance of multicarrier CDMA systems in frequencyselective Rayleigh fading channels. IEEE Transaction on Vehicular Technology. 1999, 48(5), pp.1584-1595, doi: 10.1109/25.790535.

[3] BINGHAM J.A.C. Multicarrier modulation for data transmission. IEEE Communications Magazine, 1990, 28(5), pp. 5-14, doi: 10.1109/35.54342.

[4] VERDU S. Multiuser detection. Cambridge: Cambridge University Press. 1995. doi: 10. 100zz7/978-1-4615-2251-5_6 .

[5] BRUNNEL L. Multiuser detection techniques using maximum likelihood sphere decoding in multicarrier CDMA systems. IEEE Transactions on Wireless Communications. 2004, 3(3), pp. 949-957, doi: 10.1109/twc.2004.827742.

[6] IBARS C., BAR Y. Comparing the performance of coded multiuser OFDM and coded MC-CDMA over fading channels. In: Proceedings of the IEEE International Conference on Global Telecommunications Conference. 2001, pp. 881-885. doi: 10.1109/glocom. 2001. 965545 .

[7] ABDElKader T., MOKHTAR K., ABDELAZIZ O. New Space-Time coding for Joint Blind Channel Estimation and Data Detection through Time-Varying MIMO Channels. International Journal of Computer Science Issues. 2014, 11(5), pp. 1-33. Available from: https://www. semanticscholar.org/paper/.

[8] ABUthiniEn. M. CHEN. S., WOLFGANG. A., HANZO, L. Joint maximum likelihood channel estimation and data detection for MIMO systems. In IEEE International Conference on Communications. 2007, pp. 5354-5358. doi: 10.1109/icc.2007.886.

[9] MEZGHANI A., SWINDLE H.A.L. (2016). Blind Estimation of Sparse Multi-User Massive MIMO Channels. In WSA 21th International ITG Workshop on Smart Antennas. 2017, pp. 1-5. Available from: https://arxiv.org/abs/1612.00131.

[10] SEYMAN M.N., TASPINAR N. Symbol detection using the differential evolution algorithm in MIMO-OFDM systems. Turkish Journal of Electrical Engineering \& Computer Sciences. 2013, 21(1), pp. 373-380. doi: 10.3906/elk-1103-16.

[11] PIRAK C., WANG Z.J., LIU K.R., JITAPUNKUL S. Optimum power allocation for maximum-likelihood channel estimation in space-time coded MIMO systems. In Proceedings of IEEE International Conference on Acoustics Speech and Signal Processing. 2006, 4, pp. 4-6. doi: 10.1109/icassp.2006.1661033.

[12] RAUT P.W., BADIATE S.L. MIMO-future wireless communication. International Journal of Innovative Technology and Exploring Engineering, 2013, 2(5), pp. 102-106. doi: 10.1109/ TIT.2003.810646.

[13] ZUBAIR M., CHOUDHRY M.A., NAVEED A., QURESHI I.M. Joint channel and data estimation using particle swarm optimization. IEICE Transactions on Communications. 2008, 91(9), pp. 3033-3036. doi: 10.1093/ietcom/e91-b.9.3033. 


\section{Neural Network World 6/2019, 381-401}

[14] BIGUESH M., GERSHMAN A.B. Training-based MIMO channel estimation: a study of estimator tradeoffs and optimal training signals. IEEE Transactions on Signal Processing.2006, 54(3), pp. 884-893. doi: 10.1109/TSP.2005.863008.

[15] CHEN P., KOBAYASHI H. Maximum likelihood channel estimation and signal detection for OFDM systems. In IEEE International Conference on Communications. Conference Proceedings (Cat. No. 02CH37333). 2002, 3, pp. 1640-1645. doi: 10.1109/ICC.2002.997127.

[16] KHAN M. A. Multi-user detection using computational intelligence in multi-carrier communication systems, Pakistan, 2016, PhD thesis, ISRA University Islamabad. Available from: http://prr.hec.gov.pk/jspui/handle/123456789/8007?mode $\$=\$$ full.

[17] UMAIR M., KHAN M.A., SALEEM M.A. Island Genetic Algorithm based MUD for MCCDMA System, 6th IEEE International Conference on Information and Communication Technologies, Electronic ISBN: 978-1-4673-8907-5, Electronic ISBN: 978-1-4673-8907-5, INSPEC Accession Number: 16004107. 2015, pp. 7-12. doi: 10.1109/ICICT.2015.7469588.

[18] ALI N., KHAN M.A., ADEEL M., AMIR, M. Genetic Algorithm based adaptive Receiver for MC-CDMA system with variation in Mutation Operator, International Journal of Computer Science and Information Security, USA, ISSN 1947-5500. 2016, 14 (9), pp. 215-222. Available from: https://www. academia.edu/30645146.

[19] UMAIR M., KHAN M.A., SALEEM M.A. Piranha Fish Optimization for Multi-User Detection in OFDMA System, International Journal of Advanced and Applied Sciences. 2016, 3(6), pp. 35-40. Available from: http://www.science-gate.com/IJAAS.

[20] AAZHANG B., PARIS B.P., ORSAK G.C. Neural Networks for multiuser detection in code-division multiple-access communications. IEEE Transactions on Communications. 1992 40(7), pp. 1212-1222. doi: 10.1109/26.153366.

[21] BAGADI K.P., DAS S. Neural Network-based adaptive multiuser detection schemes in SDMA-OFDM system for the wireless application. Neural Computing and Applications. 2013, 23(3), pp.1071-1082. doi: 10.1007/s00521-012-1033-z.

[22] CARLIER F., NOUVEL F. Neural Networks for multi-user detection in MC-CDMA systems. In: Proceedings of the 57th IEEE International Conference on Semiannual Vehicular Technology Conference. 2003, pp. 2399-2403. doi: 10.1007/s11277-011-0462-9.

[23] BAGADI K.P., DAS S. Efficient complex radial basis function model for multiuser detection in a space division multiple access/multiple-input multiple-output-orthogonal frequency division multiplexing system. IET Communications. 2013, 7(13), pp. 1394-1404. doi: 10.1049/iet-com.2012.0688.

[24] MITRA U., POOR H.V. Neural network techniques for adaptive multiuser demodulation. IEEE Journal on Selected Areas in Communications. 1994, 12(9), pp. 1460-1470. doi: 10. $1109 / 49.339913$.

[25] ISIK Y., TASPINAR N. Parallel interference cancellation based on the neural network in CDMA systems. IEICE Transactions on Communications. 2005. 88(1), pp. 800-806. doi: 10. 1093/ietcom/E88-B.2.800.

[26] ISIK Y., TASPINAR N. The multi-user detection in a code division multiple access with an adaptive neuro-fuzzy inference system. Journal of Information Science and Engineering. 2006, 22(6), pp. 1529-1542. Available from: https://www.semanticscholar.org.

[27] ISIK Y., TASPINAR N. Multiuser detection with neural network and PIC in CDMA systems for AWGN and Rayleigh fading asynchronous channels. Wireless Personal Communications. 2007, 43(4), pp. 1185-1194. doi: 10.1007/s11277-007-9293-0.

[28] SUE B., JAE Y.A. LMS Adaptive Receiver for Uplink Space-Time Coded MC-CDMA System. In The 12th IEEE International Conference on Advanced Communication Technology (ICACT). 2010, pp. 839-843. Available from: https://ieeexplore.ieee.org/document/ 5440155.

[29] SUE B., JAE Y.A., JEONG C., KIM H.M. Fast convergent LMS adaptive receiver for MCCDMA systems with space-time block coding. IEEE Communications Letters. 2010, 14(8), pp. 737-739. doi: 10.1109/LCOMM. 2010.08.100679. 
Abbas S. et al.: Multi user detection using fuzzy logic empowered...

[30] LAMMARE, R., SAMPAIO, R. Blind adaptive MIMO receivers for space-time block-coded DS-CDMA systems in multipath channels using the constant modulus criterion. IEEE Trans. Commun. 2010. 58(1), pp. 21-27. doi: 10.1109/TCOMM.2010.01.070549.

[31] KHAN M, A., UMAIR M., CHOUDHRY M.A.S. Accelerated assistant to the sub-optimum receiver for multi-carrier code division multiple access systems. In: The International Conference on Future Technologies $\& 3$ Communication (FTCOM). 2012, pp. 26-29. Available from: https://arxiv.org/abs/1401.5934.

[32] UMAIR M., KHAN M, A., CHOUDHRY M.A.S. GA backing to STBC based MC-CDMA systems. In: 4th IEEE International Conference on Intelligent Systems, Modeling, and Simulation. 2013 pp. 503-506. doi: 10.1109/ISMS.2013.26.

[33] KHAN M.A., UMAIR M., CHOUDHRY M.A.S. GA based adaptive receiver for MC-CDMA system, Turkish Journal of Electrical Engineering \& Computer Sciences. 2015, 23(Sup.1), pp. 2267-2277. doi: 10.3906/elk-1303-202.

[34] KHAN M.A., UMAIR M., CHOUDHRY M.A.S. Island differential evolution based adaptive receiver for MC-CDMA system. In IEEE International Conference on Information and Communication Technologies. 2015, pp. 1-6. doi: 10.1109/ICICT.2015.7469589.

[35] KHAN M.A., NASIR A., UMAIR M., ABBAS S. Time Complexity of TOMPSO Algorithm. 16th International Conference on Statistical Sciences: Advances in Statistics and Data Management: Its Role in National Growth and Socio-Economic Developments. 2018, pp. 89-100. doi: 10.1007/978-3-540-68721-4_1.

[36] ASAdUUlah M., KHAN M.A., ABBAS S., ATHAR A., RAZA S.S., AHMAD G. Blind channel and data estimation using fuzzy logic-empowered opposite learning-based mutant particle swarm optimization. Computational Intelligence and Neuroscience. 2018, 2018, pp. 1-12. doi: 10.1155/2018/6759526.

[37] KHAN M.A., UMAIR M., CHOUDHRY M.A.S., ALI M.N., ABBAS S. CDE using improved opposite based swarm optimization for MIMO systems. Journal of Intelligent \& Fuzzy Systems. 2019, 37(1), pp. 687-692. doi: 10.3233/JIFS-181127.

[38] TARIQ Z.B., KHAN M.A., ABBAS S., FATIMA A. Complexity Analysis of DE based CEUD MIMO System. 2nd IEEE International Conference on Computing, Mathematics and Engineering Technologies (iCoMET). 2019, pp. 1-5. doi: 10.1109/ICOMET.2019.8673462.

[39] IQBAL K., KHAN M. A., ABBAS S., HASAN Z. Time complexity analysis of GA-based variants uplink MC-CDMA system. SN Applied Sciences. 2019, 1(9), pp. 953-957. doi: 10. 1007/s42452-019-0984-4.

[40] ASIF M., KHAN M.A., ABBAS S., SALEEM M. Analysis of Space \& Time Complexity with PSO Based Synchronous MC-CDMA System. In: 2nd IEEE International Conference on Computing, Mathematics and Engineering Technologies (iCoMET). 2019, pp. 1-5. doi: 10. 1109/ICOMET. 2019.8673401.

[41] BAGADI K.P. Design of MC-CDMA receiver using radial basis function network to mitigate multiple access interference and nonlinear distortion. Neural Computing and Application. 2019, 31(1), pp. 1263-1273. doi: 10.1007/s00521-017-3127-0.

[42] VIVEK M.U., CHALLA N.R., BAGADI K. Lenstra Lenstra Lovász (LLL) Assisted Likelihood Ascent Search (LAS) Algorithm for Signal Detection in Massive MIMO. In IEEE International Conference on Vision Towards Emerging Trends in Communication and Networking (ViTECoN). 2019, pp. 1-4. doi: 10.1109/ViTECoN.2019.8899594. 